\section{Autophosphorylation of the DNA-dependent protein kinase catalytic subunit is required for rejoining of DNA double-strand breaks}

\author{
Doug W. Chan, ${ }^{1,2,5}$ Benjamin Ping-Chi Chen,,5 \\ Sheela Prithivirajsingh, ${ }^{3}$ Akihiro Kurimasa, ${ }^{1,4}$ \\ Michael D. Story, ${ }^{3}$ Jun Qin, ${ }^{2}$ and David J. Chen ${ }^{1,6}$ \\ ${ }^{1}$ Life Sciences Division, Lawrence Berkeley National \\ Laboratory, Berkeley, California 94720, USA; ${ }^{2}$ Verna and \\ Marrs McLean Department of Biochemistry and Molecular \\ Biology, and Department of Molecular and Cellular Biology, \\ Baylor College of Medicine, Houston, Texas 77030, USA; \\ ${ }^{3}$ Department of Experimental Radiotherapy, M. D. Anderson \\ Cancer Center, University of Texas, Houston, Texas 77030, \\ USA
}

Nonhomologous end-joining (NHEJ) is the predominant pathway that repairs DNA double-strand breaks (DSBs) in mammalian cells. The DNA-dependent protein kinase (DNA-PK), consisting of Ku and DNA-PK catalytic subunit (DNA-PKcs), is activated by DNA in vitro and is required for NHEJ. We report that DNA-PKcs is autophosphorylated at Thr2609 in vivo in a Ku-dependent manner in response to ionizing radiation. Phosphorylated DNA-PKcs colocalizes with both $\gamma$-H2AX and 53BP1 after DNA damage. Mutation of Thr2609 to Ala leads to radiation sensitivity and impaired DSB rejoining. These findings establish that Ku-dependent phosphorylation of DNA-PKes at Thr2609 is required for the repair of DSBs by NHEJ.

Received June 13, 2002; revised version accepted July 25, 2002.

The repair of DNA double-strand breaks (DSBs) in the genome is critical for the survival of cells exposed to ionizing radiation (IR; van Gent et al. 2001). Homologous recombination (HR) and nonhomologous end-joining (NHEJ) are the two principal pathways that mediate the repair of DNA DSBs in eukaryotes. HR requires the presence of a homologous chromosome or sister chromatid and is mediated by the gene products of the Saccharomyces cerevisiae RAD52 epistasis group or their homologs in other eukaryotic cells (Haber 2000), but NHEJ does not depend on the presence of homologous DNA sequences and requires both the DNA-dependent protein

[Keywords: nonhomologous end-joining; DNA damage response; autophosphorylation; DNA-PK; DNA-PKcs; Ku; radiation sensitivity; ionizing radiation]

${ }^{4}$ Present address: Life Science Division, Tottori University, Tottori 6838503, Japan.

${ }^{5}$ These authors contributed equally to this work.

${ }^{6}$ Corresponding author.

E-MAIL djchen@lbl.gov; FAX (510) 486-6816.

Article and publication are at http://www.genesdev.org/cgi/doi/10.1101/ gad.1015202. kinase complex (DNA-PK) and the XRCC4/DNA ligase IV complex (Critchlow and Jackson 1998).

DNA-PK is composed of the Ku heterodimer (which consists of the Ku70 and Ku80 subunits) and the catalytic subunit (DNA-PKcs; Smith and Jackson 1999). DNA-PKcs is a member of the phosphatidylinositol-3 (PI-3)-like kinase family that includes ATM (ataxia-telangiectasia-mutated) and ATR (ATM-Rad3-related; Durocher and Jackson 2001). Ku binds to DNA ends with very high affinity and is thought to function as the DNAbinding and regulatory subunit that stimulates DNAPKcs kinase activity (Gottlieb and Jackson 1993; Dynan and Yoo 1998). Although the biochemical properties of DNA-PK have been extensively studied in vitro, it is still not clear how it functions in vivo in the context of NHEJ. Complementation of a hamster cell line (V3) that is defective for DNA-PKcs with a wild-type human $D N A-P K c S$ cDNA rescued its radiation sensitivity and restored its DSB rejoining capacity. However, a kinasedead form of DNA-PKcs failed to rescue either defect, thus showing that the kinase activity of DNA-PK is required for the repair of DSBs by the NHEJ pathway (Kurimasa et al. 1999).

Neither the mechanism by which DNA-PK becomes activated in response to DNA damage nor its physiological targets is known. Possible targets of DNA-PK include the Wrn helicase (Yannone et al. 2001) and DNA-PKcs itself (Chan and Lees-Miller 1996), but the significance of phosphorylation of these putative targets is not known. Here we report evidence that DNA-PKcs is regulated by autophosphorylation of Thr2609 in response to IR. By virtue of colocalization with $\gamma$-H2AX and 53BP1, we show that phosphorylated DNA-PKcs is localized to sites of DNA DSBs. Furthermore, cellular studies revealed that an Ala substitution at position 2609 significantly reduces both DSB rejoining and cell survival. Thus, autophosphorylation of DNA-PKcs at Thr2609 is an important event in the repair of DSBs by the NHEJ pathway.

\section{Results and Discussion}

We previously showed that DNA-PK is capable of autophosphorylating $\mathrm{Ku} 70, \mathrm{Ku} 80$, and DNA-PKcs in vitro. Autophosphorylation of DNA-PKcs results in dissociation from $\mathrm{Ku}$ and loss of kinase activity and thus has been hypothesized to be an important regulatory mechanism (Chan and Lees-Miller 1996). To investigate the biological significance of DNA-PKcs autophosphorylation, we first identified in vitro autophosphorylation sites by mass spectrometry (Zhang et al. 1998). Highly purified DNA-PKcs and Ku were incubated in the presence of sheared calf thymus DNA and low concentrations of ATP $(50 \mu \mathrm{M})$ to allow for autophosphorylation of the most preferred site(s). Phosphorylated DNA-PKcs was analyzed by mass spectrometry, and Thr2609 was unambiguously identified as a site of autophosphorylation (Fig. 1a). Thr2609 lies in a region of DNA-PKcs that is not conserved among the various members of the PI-3 kinase family. However, Thr2609 is conserved in all known DNA-PKcs homologs (Fig. 1b), suggesting that phosphorylation of DNA-PKcs at this residue may be evolutionarily conserved. We designed a 13-residue peptide that corresponded to this conserved sequence but 
a

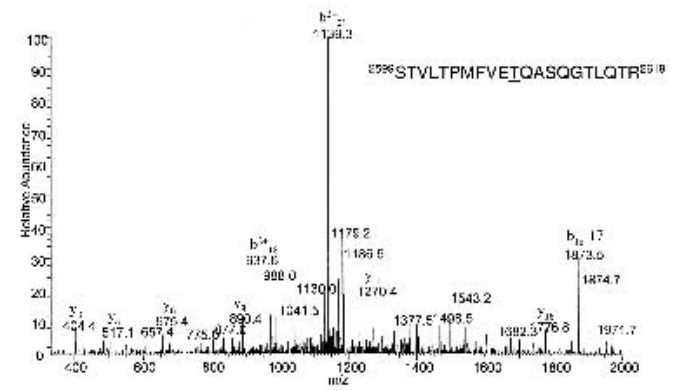

b
Himan
Dog
Honse
Mouse
Xenopus
Chicken

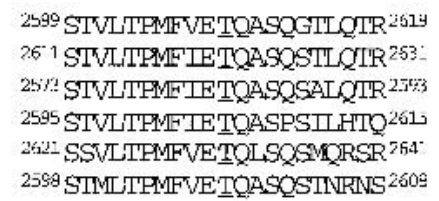

Figure 1. DNA-PKcs phosphorylation of Thr2609. (a) Mass spectrometry (MS) identification of the DNA-PKcs in vitro autophosphorylation site. MS spectra of the identified tryptic-phosphopeptide; the underlined T corresponds to Thr2609 and is the site of phosphorylation. (b) Alignment of human DNA-PKcs Thr2609 with DNA-PKcs sequences available in the National Center for Biotechnology Information database.

contained a phospho-threonine at the 2609 position, used it to raise a rabbit polyclonal antibody and then affinity-purified an antibody recognizing the phosphorylated Thr2609 (pT2609Ab). To confirm the specificity of the pT2609Ab, we expressed and purified GST DNAPKcs fragments spanning residues 2500-2700 that contained the wild-type sequence or with an Ala point mutant at position 2609. The GST fragments were in vitro phosphorylated with purified DNA-PK and analyzed by Western blotting with pT2609Ab (Fig. 2a, top) or against GST (Fig. 2a, bottom). The pT2609Ab cross-reacted with the phosphorylated wild-type GST fragment, but not with the fragment containing the T2609A mutation (Fig. $2 \mathrm{a}$, top). Furthermore, immunoblotting with the pT2609Ab of unphosphorylated DNA-PKcs at 100-fold molar excess (relative to an amount of phosphorylated DNA-PKcs that was readily detectable) did not produce any detectable signal (Fig. 2b, cf. lanes 2 and 9).

Exponentially growing HeLa cells were treated with IR and analyzed with the pT2609Ab to investigate Thr2609 phosphorylation in vivo (Fig. 2c,d). Phosphorylation of Thr2609 is inducible with IR and can be detected as early as $10 \mathrm{~min}$ after treatment with $10 \mathrm{~Gy}$ (Fig. 2c). At this dose, the phosphorylation of Thr2609 reaches a maximum after 30 min and persists for up to $4 \mathrm{~h}$, becoming undetectable by $6 \mathrm{~h}$ after IR (Fig. 2c). It has been previously shown that DNA-PK functions in the initial fast phase of the biphasic repair of DSBs, such that within the first hour after damage, $\sim 80 \%$ of DSBs are rejoined in a DNA-PK-dependent manner (DiBiase et al. 2000). The kinetics of Thr2609 phosphorylation that we have observed (Fig. 2c) correlate very closely with the model proposed by DiBiase et al. (2000) and suggest that phosphorylation of this site in response to DNA damage could be pertinent to rejoining of DSBs. Phosphorylation of Thr2609 is also dependent on the dose and can be induced with $2 \mathrm{~Gy}$; at $10 \mathrm{~Gy}$, detection with the pT2609Ab reached saturation (Fig. 2d). Because phosphorylation of Thr2609 can be observed with a dose as low as $2 \mathrm{~Gy}$, these results suggest that phosphorylation of DNA-PKcs is very sensitive to the presence of DSBs in the genome. We have also observed phosphorylation of Thr2609 in response to IR in a lymphoblastoid cell line (Jurkat), in a glioma cell line (M059K), and in primary human fibroblasts (data not shown), and thus, this event appears to be a general phenomenon that is not cell-type specific.

The activity of the PI-3 kinase family members, including DNA-PK, can be inhibited by wortmannin (Sarkaria et al. 1998). Treatment of HeLa cells with 20 $\mu \mathrm{M}$ wortmannin did not alter the DNA-PKcs protein levels (Fig. 3a, bottom); however, the wortmannin treatment resulted in a significant decrease in the phosphorylation of Thr2609 after 10 Gy IR (Fig. 3a, top), suggesting that phosphorylation of Thr2609 in response to DNA damage is mediated by a PI-3 kinase. It has been previously shown that $20 \mu \mathrm{M}$ wortmannin inhibits DNA-PKmediated repair of DNA DSBs and increases the sensitivity to radiation (Chernikova et al. 1999; DiBiase et al. 2000). However, at this concentration it may also inhibit ATM, another PI-3 kinase that is also activated by ionizing radiation (Banin et al. 1998; Canman et al. 1998). To distinguish between these possibilities, a human A-T cell line that was complemented with vector (Fig. 3b, lanes 1,2 ) or with full-length wild-type ATM cDNA (Fig. $3 \mathrm{~b}$, lanes 3,4$)$ was treated with 10 Gy IR and analyzed using the pT2609Ab (Fig. 3b, top) and an anti-DNA-PKcs monoclonal antibody (Fig. 3b, bottom). IR-induced phosphorylation of DNA-PKcs at Thr2609 was observed in
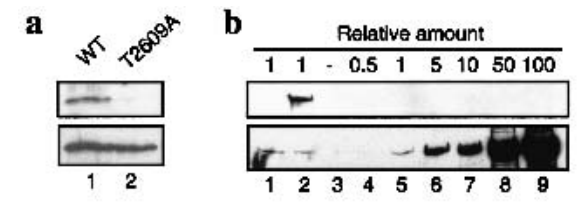

c

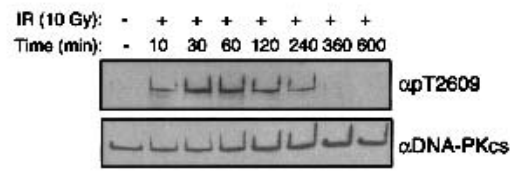

d

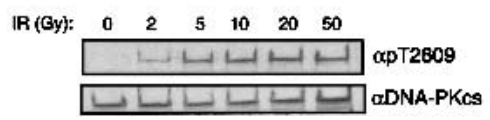

Figure 2. Characterization of Thr2609 phosphorylation. (a) Specificity of pT2609Ab. GST fragments spanning amino acids 25002700 that contain either the wild-type DNA-PKcs sequence or a T2609A point mutation were in vitro phosphorylated with purified DNA-PK and analyzed with the pT2609Ab (top) and anti-GST (bottom) to show equal loading. (b) pT2609Ab does not recognize unphosphorylated DNA-PKcs. Western blotting with pT2609Ab (top) and 25-4 DNA-PKcs monoclonal antibody (bottom) with mock or autophosphorylated DNA-PKcs (lanes 1 and 2, respectively) and with purified, unphosphorylated DNA-PKcs (lanes 3-9) at the indicated molar ratios relative to the amount of protein in lanes 1,2. (c) Kinetics of Thr 2609 phosphorylation in vivo. HeLa cells were either mock-treated or irradiated with $10 \mathrm{~Gy}$ and allowed to recover for the indicated times. Nuclear extracts were Western blotted with pT2609Ab (top), and then blots were stripped and reprobed with the 25-4 DNA-PKcs monoclonal antibody (bottom). (d) Dose dependence of Thr 2609 phosphorylation. HeLa cells were irradiated with the indicated dose of ionizing radiation and allowed to recover for 30 min. Nuclear extracts were first analyzed by Western blot with pT2609Ab (top) and then with 25-4 monoclonal to show equal loading (bottom). 
a
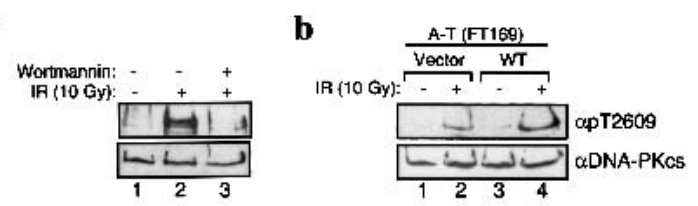

c KURO SIRNA:

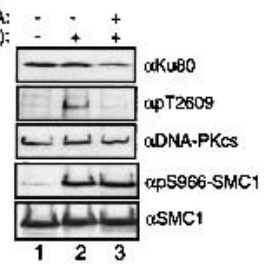

d
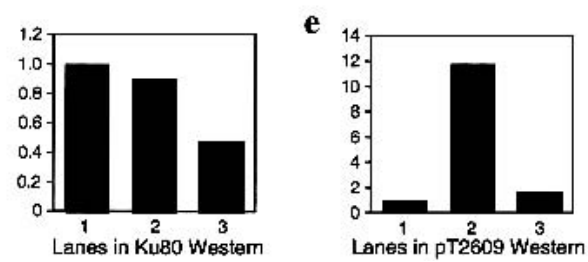

Figure 3. Characterization of Thr2609 phosphorylation in vivo. $(a)$ Inhibition of Thr2609 phosphorylation by wortmannin. HeLa cells were preincubated with DMSO (dimethyl sulfoxide; lanes 1,2) or 20 $\mu M$ wortmannin (lane 3) for 30 min before treatment with 10 Gy of ionizing radiation (IR; lanes 2,3). Nuclear extracts were prepared 30 min after IR and subsequently analyzed for Thr2609 phosphorylation (top) and with 25-4 monoclonal (bottom). (b) Phosphorylation of Thr2609 is ATM-independent. A-T cells (FT169) complemented with vector $($ lanes 1,2) or full-length wild-type ATM cDNA (lanes 3,4 ) were treated with 10 Gy of IR. Nuclear extracts were analyzed for Thr2609 phosphorylation (top) and DNA-PKcs levels (bottom). (c) Phosphorylation of Thr2609 is Ku-dependent. HeLa cells were mock-transfected (lanes 1,2) or transfected with a short interfering Ku80 RNA (lane 3) for $96 \mathrm{~h}$ before irradiation with $10 \mathrm{~Gy}$ of IR. Nuclear extracts were analyzed by Western blotting with the indicated antibodies. $(d)$ Quantification of Ku80 Western signal from $c$. Values reported are relative to the signal observed in lane 1 and assigned an arbitrary value of one. (e) Quantification of pT2609Ab signal from $c$. Values reported are relative to the signal observed in lane 1.

both cell lines (Fig. 3b), although the extent of phosphorylation is somewhat reduced in the vector-complemented cells. This result establishes that phosphorylation of DNA-PKcs at Thr2609 in response to damage does not require ATM and that Thr2609 of DNA-PKcs is therefore unlikely to be a direct target for the ATM kinase. However, an indirect contribution of ATM-dependent signaling cannot be ruled out.

In vitro, Ku stimulates the kinase activity of DNAPKcs (Dvir et al. 1992; Gottlieb and Jackson 1993). However, the role of $\mathrm{Ku}$ in activating DNA-PKcs in vivo is not known. Several studies have suggested that DNAPKcs by itself is capable of binding to DNA ends (Cary et al. 1997; Yaneva et al. 1997) and that it possesses kinase activity in the absence of Ku (Yaneva et al. 1997; Hammarsten and Chu 1998). To address the issue of a requirement for $\mathrm{Ku}$ in phosphorylation of DNA-PKes at Thr2609, we used short interfering RNA (siRNA) to reduce the levels of $\mathrm{Ku}$ in HeLa cells (Elbashir et al. 2001). Transient transfection of HeLa cells with a 21-bp siRNA that is specific to Ku80 reduced the Ku80 protein level to $\sim 50 \%$ of that in the mock-transfected cells (Fig. 3c,d) without affecting the DNA-PKcs protein level (Fig. 3c). Strikingly, these Ku80 knockdown cells showed an $\sim 80 \%$ reduction in DNA-PKcs Thr2609 phosphorylation in response to $10 \mathrm{~Gy}$ of IR (Fig. 3c,e). The decrease in Ku80 protein does not have a global effect on the DNA damage response pathway, because phosphorylation of the structural maintenance of chromosome protein 1 (SMC1) at Ser966, a site that is phosphorylated by ATM in response to IR (Kim et al. 2002; Yazdi et al. 2002), is normal in Ku80 knockdown cells (Fig. 3c). These results support an in vivo requirement for $\mathrm{Ku}$ in the activation of DNA-PKcs kinase. Taken together with the fact that Thr2609 was initially identified as a target for autophosphorylation of DNA-PKcs in vitro and that in vivo its phosphorylation is wortmannin-sensitive but does not require ATM, the phosphorylation of Thr2609 in DNAPKcs in response to DNA damage is most likely mediated by DNA-PKcs itself is strongly supported.

Current models for the function of DNA-PK in NHEJ imply that it must be located at the site of the DSB (Smith and Jackson 1999), although it has been difficult to show this directly because of the high levels of DNAPK in mammalian cells. However, if IR-induced phosphorylation of DNA-PKcs has functional significance, the phosphorylated DNA-PKcs should localize to sites of DSB. To test this prediction, we examined DNA-PKcs localization in the cell by immunofluorescence microscopy. As expected, immunostaining of DNA-PKcs in primary human fibroblasts with the specific monoclonal antibody (25-4) produced very strong signals throughout the nucleus in both unirradiated and irradiated cells (Fig. 4a, red). In contrast, immunostaining with the pT2609Ab revealed both increased signal and formation of foci in response to exposure to 5 Gy IR (Fig. 4a, green), confirming that phosphorylation of Thr2609 is inducible by DNA damage. We also examined immunostaining with both antibodies in two human glioma cell lines that differ in their DNA-PKcs status (Lees-Miller et al. 1995). In the DNA-PKcs-deficient M059J cells, no signal was detectable with either antibody at $30 \mathrm{~min}$ after exposure to 5 Gy IR (Fig. 4b), confirming the lack of DNA-PKcs protein in this cell line. In contrast, immunostaining with the 25-4 monoclonal antibody in the DNA-PKcsproficient M059K cell line produced intense signal throughout the nucleus. More importantly, staining with the pT2609Ab again clearly revealed foci formation in response to DNA damage (Fig. 4b). The lack of detectable foci in M059J cells confirms the specificity of the pT2609Ab and shows that the foci observed with this antibody in DNA-PKcs-proficient cells do indeed correspond to the phosphorylated form of DNA-PKcs.

To address whether phosphorylated DNA-PKcs is associated with DSBs after IR, we examined its possible colocalization with the phosphorylated histone H2AX $(\gamma-\mathrm{H} 2 \mathrm{AX})$ and the p53-binding protein 1 (53BP1). $\gamma$-H2AX foci have been previously shown to form almost immediately after exposure to IR and localize at sites of DNA strand breaks (Rogakou et al. 1999; Paull et al. 2000). 53BP1 has also been shown to localize to sites of damage by virtue of its colocalization with $\gamma-\mathrm{H} 2 \mathrm{AX}$ and is proposed to play an important role in the ATM-mediated DNA damage-signaling pathway (Rappold et al. 2001). Primary human fibroblasts were treated with IR, incubated for $30 \mathrm{~min}$ after IR, and then fixed and stained with both pT2609Ab and antibodies to either $\gamma$-H2AX (Fig. 4c) or 53BP1 (Fig. 4d). All three antibodies revealed formation of IR-induced foci, and the merged images show strong colocalization of DNA-PKcs phosphorylated at 
Chan et al.

a
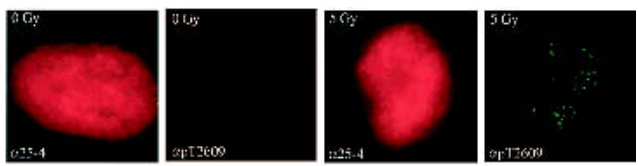

b
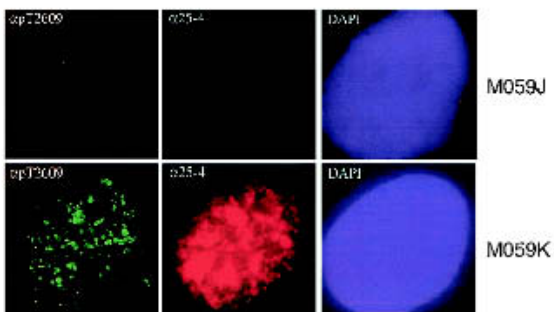

c
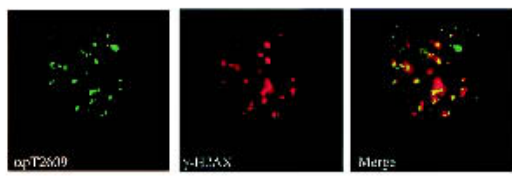

d
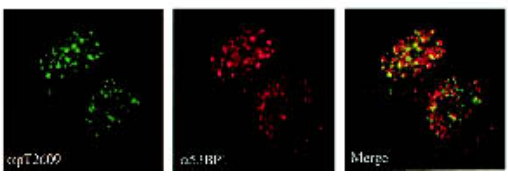

Figure 4. DNA-PKcs forms foci in response to ionizing radiation (IR). (a) DNA damage-induced pT2609 foci. Primary human fibroblasts were mock-treated or irradiated with 5 Gy of IR (as indicated) and allowed to recover for $30 \mathrm{~min}$ before fixation and staining with 25-4 monoclonal to DNA-PKcs (red) and pT2609Ab (green). (b) pT2609 foci are absent in DNA-PKcs-deficient cells. The DNAPKcs-deficient (M059J) and the DNA-PKcs-proficient (M059K) cell lines were irradiated with $5 \mathrm{~Gy}$ and allowed to recover for $30 \mathrm{~min}$ before immunostaining with 25-4 monoclonal (red) and pT2609Ab (green) antibodies. (c) Colocalization of phosphorylated DNA-PKcs with $\gamma$-H2AX. Human primary fibroblasts were irradiated with 1 Gy, fixed at 30 min after IR, and stained with pT2609Ab (green) and monoclonal antibody against phosphoSer139 of histone H2AX (red; Upstate Biotech). The two images were merged, and colocalization of pT2609 and $\gamma$-H2AX foci are depicted as yellow. (d) Colocalization of phosphorylated DNA-PKcs with 53BP1. The experiment was conducted as in $c$ but using 53BP1 monoclonal (red).

Thr2609 with both $\gamma$-H2AX and 53BP1 (Fig. 4c and d, respectively). These results provide the first evidence that DNA-PKcs is indeed localized to sites of DSBs in the cell and thus is at the right location to play a direct role in DSB repair.

To directly investigate the biological significance of DNA-PKcs phosphorylation at Thr2609 in relation to rejoining of DSBs, we expressed wild-type or mutant (T2609A) DNA-PKcs in the V3 CHO cell line that is defective in DNA-PKcs (Kurimasa et al. 1999). Stable V3 cell lines expressing either wild-type (V3-F18) or T2609A-mutant proteins (V3-T2609A1) were isolated. The V3 cell line transfected with the vector alone (V3JM) contained undetectable levels of DNA-PKcs protein, whereas the V3-F18 and mutant V3-T2609A1 cells showed similar levels of expression of the DNA-PKcs protein (Fig. 5a). The radiation sensitivity and DNA DSB rejoining capacity of these stably transfected cell lines was investigated.

The radiation sensitivity of these cell lines was examined by assaying for their colony-forming ability after IR. As we previously showed, complementation of V3 cells by expression of wild-type human DNA-PKcs substantially increased their radioresistance (Fig. 5b), resulting in survival levels comparable to those of wild-type $\mathrm{CHO}$ cells (Kurimasa et al. 1999). In contrast, although expression of the T2609A mutant protein improved the survival of the V3-T2609A1 cell line, it is significantly lower than what was observed for V3-F18. The dose of IR required for $10 \%$ survival of the V3-JM, V3-T2609A1, and V3-F18 cell lines was $1.2 \mathrm{~Gy}, 2.4 \mathrm{~Gy}$, and $5 \mathrm{~Gy}$, respectively (Fig. 5b). Thus, the $\mathrm{D}_{10}$ value is approximately fourfold higher for V3-F18 compared with the V3-JM noncomplemented cells (i.e., $5 \mathrm{~Gy} / 1.2 \mathrm{~Gy}$, whereas resistance at the $10 \%$ survival level was increased by only twofold in the V3-T2609A1 cells (5Gy/ $2.4 \mathrm{~Gy})$. Therefore, phosphorylation of Thr2609 is important for cell viability in response to IR. In addition, the FAR (fraction of activity released) assay was used to evaluate the ability of each of the three cell lines to rejoin DSBs induced by IR. The FAR assay uses pulsed field gel electrophoresis to indirectly measure the intactness

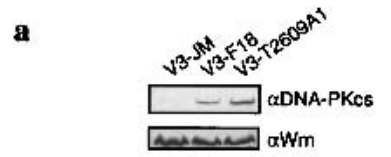

b
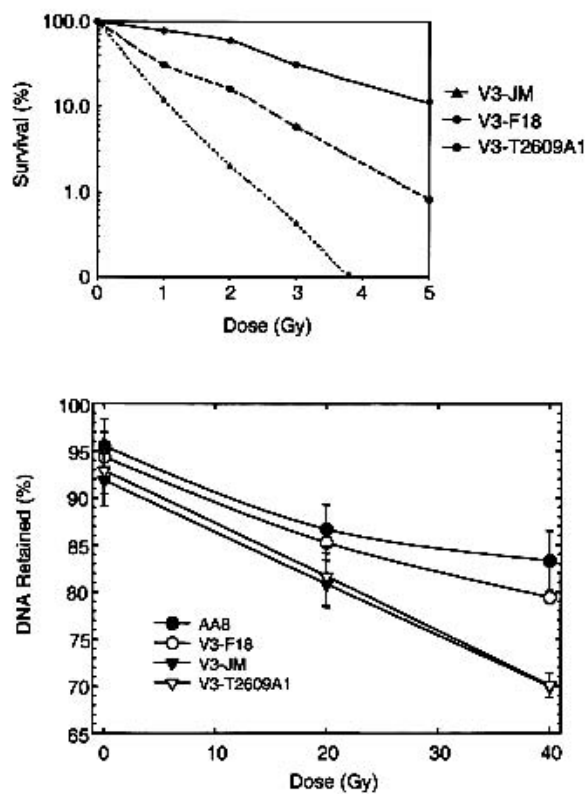

Figure 5. Phosphorylation of Thr2609 is required for double-strand break (DSB) repair. (a) Protein expression levels in V3-complemented cells. Nuclear extracts prepared from V3 transfected with vector alone (V3-JM), full-length wild-type DNA-PKcs (V3-F18), or DNA-PKcs containing the T2609A point mutant (V3-T2609A1) were analyzed for DNA-PKcs protein expression levels (top). Hamster Werner proteins were analyzed to show equal sample loading (bottom). (b) The colony formation assay was performed to compare radiation sensitivity of the three transfected V3 cell lines. V3-JM, V3-F18, and V3-T2609A1 were irradiated at the indicated doses and plated for analysis of survival and colony-forming ability. (c) FAR (fraction of activity released) assay to measure the presence of DNA DSBs. The V3-JM, V3-F18, and V3-T2609A1 cell lines and the parental CHO cell line (AA8) were irradiated at the indicated dose and analyzed for the presence of DSBs by the FAR assay. The greater the percentage of DNA retained, the fewer the number of DSBs remained. 
of DNA in cells after gentle lysis in agarose plugs by quantifying the amount of DNA released from the wells immediately after IR exposure as a function of dose or after a period of incubation to allow repair after a given dose (Story et al. 1994). The V3-F18 and the parental AA8 CHO cell lines showed comparable DSB rejoining capacities (Fig. 5c, open and closed circles, respectively). In contrast, the V3-JM and the V3-T2609A1 cells were significantly more defective in the rejoining of DNA DSBs at $4 \mathrm{~h}$ after irradiation (Fig. 5c), consistent with our previous observations (Kurimasa et al. 1999) and with the hypothesis that DNA-PKcs plays an important role in repair of DSBs. Together, these results provide compelling evidence that phosphorylation of DNA-PKcs at Thr2609 is important for rejoining of DSBs and for cell survival in response to DNA damage caused by IR.

In summary, we have identified Thr2609 as a DNA damage-inducible phosphorylation site. We have shown that phosphorylated DNA-PKcs plays important roles in the repair of DNA DSBs, by virtue of its localization at the site of damage and because cells expressing the DNA-PKes protein that contains the T2609A point mutant show an increase in radiation-sensitivity and an impaired capacity to rejoin DNA DSBs. It is very likely that Thr2609 is not the only (auto)phosphorylation site on DNA-PKcs, because phosphoamino acid analysis of DNA-PKcs that is in vivo labeled with ${ }^{32} \mathrm{P}$ reveals the presence of both phosphothreonine and phosphoserine residues (D.W. Chan and D.J. Chen, unpubl.). The presence of additional DNA-PKcs phosphorylation sites may explain why the V3-T2609A1 cells showed only a roughly twofold increase in radiation sensitivity (at 10 Gy; Fig. 5b). We speculate that in response to DNA damage, phosphorylation of multiple sites may be required for proper DNA-PK function, and thus, mutation of Thr2609 produced only a twofold increase in radiation sensitivity.

Three large PI-3-like protein kinases-ATM, ATR, and DNA-PKcs-play important roles in the maintenance of the human genome (Critchlow and Jackson 1998). ATM and ATR have been firmly established as central players in the activation of checkpoint pathways that relay the DNA damage signal to downstream effectors through phosphorylation (Zhou and Elledge 2000). Many of the components of these pathways have been elucidated. In contrast, DNA-PKcs activity appears to not function in the checkpoint pathways but instead to function in the repair process itself. In comparison to ATM and ATR, very little is known about the mechanism of DNA-PK action in response to DNA damage. Our findings that DNA-PKcs autophosphorylation at Thr2609 in response to IR establishes its role in DSB repair. Additionally, determining that phosphorylation is detectable within 10 min after IR and that the phosphorylated protein is found in association with $\gamma$-H2AX and 53BP1 proteins in the vicinity of DSBs in the cell at early times after IR provide important clues to this mechanism. Although the precise role of Thr2609 phosphorylation is not known, possibilities include regulation of the kinase activity of DNA-PKcs or in mediating the protein-protein interactions for recruitment of other repair factors that coordinate DSB rejoining by NHEJ. Both hypotheses are consistent with our previous observations from in vitro studies that DNA-PKcs phosphorylation results in its dissociation from $\mathrm{Ku}$ and loss of its kinase activity (Chan and Lees-Miller 1996). Future research to identify pos- sible additional sites of phosphorylation in DNA-PKcs, additional DNA-PK substrates, and proteins that interact with DNA-PKcs in response to DNA damage will aid in elucidating the molecular mechanism of the DNAPK-dependent NHEJ pathway.

\section{Materials and methods}

Identification of DNA-PKcs autophosphorylation site

Purified human DNA-PKcs and Ku were autophosphorylated as previously described (Chan and Lees-Miller 1996) with the following changes: $50 \mu \mathrm{M}$ ATP was used instead of $250 \mu \mathrm{M}$, and the phosphorylated DNAPKcs band was excised, digested with trypsin, and analyzed by mass spectrometry as previously described (Zhang et al. 1998).

\section{Antibodies}

pT2609Ab polyclonal antibodies were prepared by immunizing New Zealand white rabbits with KLH (keyhole limpet hemacyanin)-conjugated phosphopeptide TPMFVET[PO3]QASQGT. The phosphospecific antibodies were affinity-purified through a phosphopeptide-conjugated Sepharose CL-4B column. Eluted IgGs were then passed through the corresponding unphosphorylated peptide column to deplete any IgGs that were not specific to $\mathrm{pThr2609}$. DNA-PKcs 25-4 monoclonal antibody was purchased from NeoMarkers. $\gamma$-H2AX monoclonal antibody was purchased from Upstate Biotech. 53BP1 monoclonal antibody was a generous gift from Dr. Junjie Chen (Mayo Clinic, Rochester, MN). Polyclonal antibodies to phospho-Ser966 of SMC1 were described in Yazdi et al. (2002).

\section{Cell extracts and Western blot analysis}

Nuclear extracts (P10) were prepared as described (Lees-Miller et al. 1990); 20 to $60 \mu \mathrm{g}$ of each sample was analyzed by Western blotting as described (Chan et al. 1996). For analysis of the V3 cell lines, the DNAcellulose pull-down method of Finnie et al. (1995) was used to first concentrate DNA-PKcs onto the cellulose and then subject it to SDS-PAGE for Western blotting.

Immunofluorescence

Immunofluorescence was performed as previously described in Burma et al. (2001).

Site-directed mutagenesis and isolation of the mutant cell lines. A 3-kb HindIII fragment of DNA-PKcs cDNA covering Thr2609 was used as the template for generating the T2609A mutation of DNA-PKcs cDNA. Site-directed mutagenesis was performed using the Quik Change site-directed mutagenesis kit (Stratagene) and the forward (TCCGAT GTTTGTGGAGGACCAGGCCTCCCAGGGC) and reverse (GCCCTG GGAGGCCTGGTCCTCCACAAACATCGGA) primers. The mutated DNA-PKcs cDNA fragment was assembled back into the full-length DNA-PKcs cDNA as described previously (Kurimasa et al. 1999). T2609A DNA-PKcs expression plasmid together with pSV2 neo plasmid was transfected into the V3 cell line. Forty-eight hours after transfection, cells were replated on selection medium containing $400 \mu \mathrm{g} / \mathrm{mL}$ of G418 for 10 days. Individual colonies were isolated and further characterized for expression.

Cell survival and DNA DSB repair assays

Colony formation and FAR assays were performed as previously described (Kurimasa et al. 1999).

\section{Ku80 SiRNA}

The siRNA sequence (UACAAACAUGGUUAUCACCdTdT) that corresponds to nucleotides 139-159 of Ku80 cDNA was purchased from Dharmacon Research Inc. and prepared according to manufacturer directions. Transfection of HeLa cells was performed as described in Elbashir et al. (2001).

\section{Acknowledgments}

We thank Dr. Junjie Chen for 53BP1 monoclonal antibodies; Dr. Yossi Shiloh for A-T cells; Shun-Jen Yang and Michael Murphy for technical assistance; and Drs. Patricia Ouimet, Parvin Yazdi, and Priscilla Cooper for discussion and critical reading of the manuscript. This work was 
supported by the United States Department of Energy under contract DE-AC03-76SF00098 and by National Institutes of Health grants CA50519 and AG917709 to D.J.C. and CA92584 to J.Q. D.W.C. is supported by postdoctoral fellowships from the Canadian Institutes of Health Research (CIHR) and the Alberta Heritage Foundation for Medical Research (AHFMR). J.Q. is a recipient of a career development award from the Department of Defense Breast Cancer Research Program (DAMD17-00-1-0146).

The publication costs of this article were defrayed in part by payment of page charges. This article must therefore be hereby marked "advertisement" in accordance with 18 USC section 1734 solely to indicate this fact.

\section{References}

Banin, S., Moyal, L., Shieh, S., Taya, Y., Anderson, C.W., Chessa, L., Smorodinsky, N.I., Prives, C., Reiss, Y., Shiloh, Y., et al. 1998. Enhanced phosphorylation of p53 by ATM in response to DNA damage. Science 281: 1674-1677.

Burma, S., Chen, B.P., Murphy, M., Kurimasa, A., and Chen, D.J. 2001. ATM phosphorylates histone $\mathrm{H} 2 \mathrm{AX}$ in response to DNA doublestrand breaks. J. Biol. Chem. 276: 42462-42467.

Canman, C.E., Lim, D.S., Cimprich, K.A., Taya, Y., Tamai, K., Sakaguchi, K., Appella, E., Kastan, M.B., and Siliciano, J.D. 1998. Activation of the ATM kinase by ionizing radiation and phosphorylation of p53. Science 281: 1677-1679.

Cary, R.B., Peterson, S.R., Wang, J., Bear, D.G., Bradbury, E.M., and Chen, D.J. 1997. DNA looping by Ku and the DNA-dependent protein kinase. Proc. Nat1. Acad. Sci. 94: 4267-4272.

Chan, D.W. and Lees-Miller, S.P. 1996. The DNA-dependent protein kinase is inactivated by autophosphorylation of the catalytic subunit. $J$ Biol. Chem. 271: 8936-8941.

Chan, D.W., Mody, C.H., Ting, N.S., and Lees-Miller, S.P. 1996. Purification and characterization of the double-stranded DNA-activated protein kinase, DNA-PK, from human placenta. Biochem. Cell. Biol. 74: 67-73.

Chernikova, S.B., Wells, R.L., and Elkind, M.M. 1999. Wortmannin sensitizes mammalian cells to radiation by inhibiting the DNA-dependent protein kinase-mediated rejoining of double-strand breaks. $R a$ diat. Res. 151: 159-166.

Critchlow, S.E. and Jackson, S.P. 1998. DNA end-joining: From yeast to man. Trends Biochem. Sci. 23: 394-398.

DiBiase, S.J., Zeng, Z.C., Chen, R., Hyslop, T., Curran, Jr., W.J., and Iliakis, G. 2000. DNA-dependent protein kinase stimulates an independently active, nonhomologous, end-joining apparatus. Cancer Res. 60: 1245-1253.

Durocher, D. and Jackson, S.P. 2001. DNA-PK, ATM and ATR as sensors of DNA damage: Variations on a theme? Curr. Opin. Cell. Biol. 13: 225-231.

Dvir, A., Peterson, S.R., Knuth, M.W., Lu, H., and Dynan, W.S. 1992. Ku autoantigen is the regulatory component of a template-associated protein kinase that phosphorylates RNA polymerase II. Proc. Natl. Acad. Sci. 89: 11920-11924.

Dynan, W.S. and Yoo, S. 1998. Interaction of Ku protein and DNA-dependent protein kinase catalytic subunit with nucleic acids. Nucleic Acids Res. 26: 1551-1559.

Elbashir, S.M., Harborth, J., Lendeckel, W., Yalcin, A., Weber, K., and Tuschl, T. 2001. Duplexes of 21-nucleotide RNAs mediate RNA interference in cultured mammalian cells. Nature 411: 494-498.

Finnie, N.J., Gottlieb, T.M., Blunt, T., Jeggo, P.A., Jackson, S.P. 1995. DNA-dependent protein kinase activity is absent in xrs-6 cells: implications for site-specific recombination and DNA double-strand break repair. Proc. Natl. Acad. Sci. 92: 320-324.

Gottlieb, T.M. and Jackson, S.P. 1993. The DNA-dependent protein kinase: Requirement for DNA ends and association with $\mathrm{Ku}$ antigen. Cell 72: 131-142.

Haber, J.E. 2000. Partners and pathways repairing a double-strand break. Trends Genet. 16: 259-264.

Hammarsten, O. and Chu, G. 1998. DNA-dependent protein kinase: DNA binding and activation in the absence of Ku. Proc. Natl. Acad. Sci. 95: 525-530.

Kim, S.T., Xu, B., and Kastan, M.B. 2002. Involvement of the cohesin protein, Smc1, in Atm-dependent and independent responses to DNA damage. Genes \& Dev. 16: 560-570.

Kurimasa, A., Kumano, S., Boubnov, N.V., Story, M.D., Tung, C.S., Peterson, S.R., and Chen, D.J. 1999. Requirement for the kinase activity of human DNA-dependent protein kinase catalytic subunit in DNA strand break rejoining. Mol. Cell Biol. 19: 3877-3884.

Lees-Miller, S.P., Chen, Y.R., and Anderson, C.W. 1990. Human cells contain a DNA-activated protein kinase that phosphorylates simian virus $40 \mathrm{~T}$ antigen, mouse p53, and the human Ku autoantigen. Mol. Cell Biol. 10: 6472-6481.

Lees-Miller, S.P., Godbout, R., Chan, D.W., Weinfeld, M., Day III, R.S., Barron, G.M., and Allalunis-Turner, J. 1995. Absence of p350 subunit of DNA-activated protein kinase from a radiosensitive human cell line. Science 267: 1183-1185.

Paull, T.T., Rogakou, E.P., Yamazaki, V., Kirchgessner, C.U., Gellert, M., and Bonner, W.M. 2000. A critical role for histone H2AX in recruitment of repair factors to nuclear foci after DNA damage. Curr. Biol. 10: $886-895$.

Rappold, I., Iwabuchi, K., Date, T., and Chen, J. 2001. Tumor suppressor p53 binding protein 1 (53BP1) is involved in DNA damage-signaling pathways. J. Cell. Biol. 153: 613-620.

Rogakou, E.P., Boon, C., Redon, C., and Bonner, W.M. 1999. Megabase chromatin domains involved in DNA double-strand breaks in vivo. $J$. Cell. Biol. 146: 905-916.

Sarkaria, J.N., Tibbetts, R.S., Busby, E.C., Kennedy, A.P., Hill, D.E, and Abraham, R.T. 1998. Inhibition of phosphoinositide 3-kinase related kinases by the radiosensitizing agent wortmannin. Cancer Res. 58: 4375-4382.

Smith, G.C. and Jackson, S.P. 1999. The DNA-dependent protein kinase. Genes \& Dev. 13: 916-934.

Story, M.D., Mendoza, E.A., Meyn, R.E., and Tofilon, P.J. 1994. Pulsedfield gel electrophoretic analysis of DNA double-strand breaks in mammalian cells using photostimulable storage phosphor imaging. Int. J. Radiat. Biol. 65: 523-528.

van Gent, D.C., Hoeijmakers, J.H., and Kanaar, R.. 2001. Chromosomal stability and the DNA double-stranded break connection. Nat. Rev. Genet. 2: 196-206.

Yaneva, M., Kowalewski, T., and Lieber, M.R 1997. Interaction of DNAdependent protein kinase with DNA and with Ku: Biochemical and atomic-force microscopy studies. EMBO J. 16: 5098-5112.

Yannone, S.M., Roy, S., Chan, D.W., Murphy, M.B., Huang, S., Campisi, J., and Chen, D.J. 2001. Werner syndrome protein is regulated and phosphorylated by DNA-dependent protein kinase. J. Biol. Chem. 276: 38242-38248.

Yazdi, P.T., Wang, Y., Zhao, S., Patel, N., Lee, E.Y., and Qin, J. 2002. SMC1 is a downstream effector in the ATM/NBS1 branch of the human S-phase checkpoint. Genes \& Dev. 16: 571-582.

Zhang, X., Herring, C.J., Romano, P.R., Szczepanowska, J., Brzeska, H., Hinnebusch, A.G, and Qin, J. 1998. Identification of phosphorylation sites in proteins separated by polyacrylamide gel electrophoresis. Anal. Chem. 70: 2050-2059.

Zhou, B.B. and Elledge, S.J. 2000. The DNA damage response: Putting checkpoints in perspective. Nature 408: 433-439. 


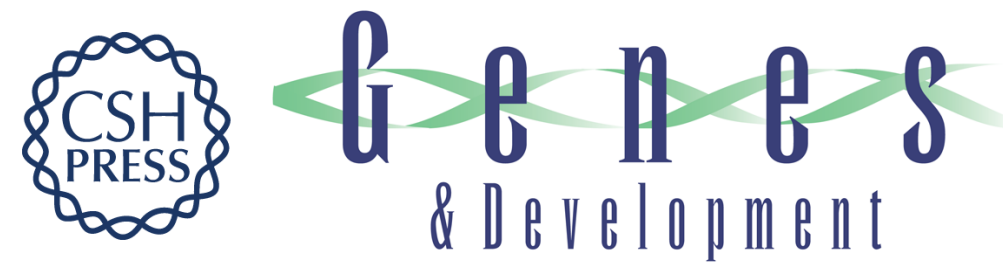

\section{Autophosphorylation of the DNA-dependent protein kinase catalytic subunit is required for rejoining of DNA double-strand breaks}

Doug W. Chan, Benjamin Ping-Chi Chen, Sheela Prithivirajsingh, et al.

Genes Dev. 2002, 16:

Access the most recent version at doi:10.1101/gad.1015202

References

This article cites 33 articles, 20 of which can be accessed free at: http://genesdev.cshlp.org/content/16/18/2333.full.html\#ref-list-1

License

Email Alerting

Receive free email alerts when new articles cite this article - sign up in the box at the top Service right corner of the article or click here.

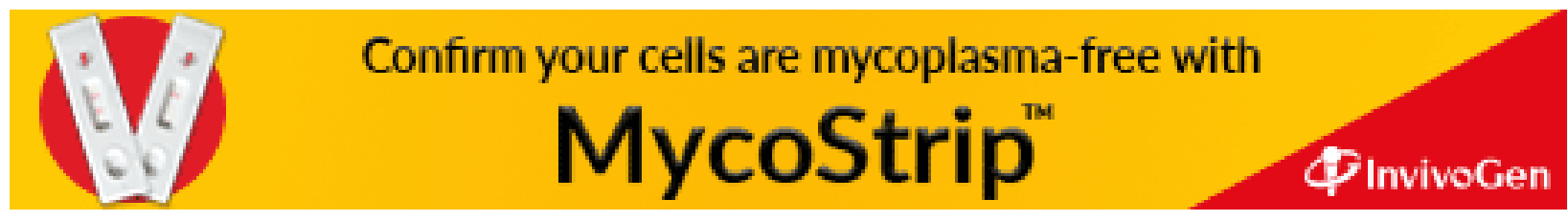

